\title{
Response strength and temporal control in fixed-interval schedules
}

\author{
RANDOLPH C. GRACE \\ University of Canterbury, Christchurch, New Zealand \\ and \\ JOHN A. NEVIN \\ University of New Hampshire, Durham, New Hampshire
}

\begin{abstract}
Pigeons responded in a two-component peak procedure in which the components differed in terms of reinforcement magnitude (Experiment 1), immediacy (Experiment 2), or probability (Experiment 3). The prediction of behavioral momentum theory that responding in the relatively richer component should be more resistant to change was tested by (1) presenting response-independent food in the intervals between components according to a variable-time (VT) schedule, (2) prefeeding, and (3) extinction. In all the experiments, peak location in baseline occurred earlier, relative to the schedule value in the richer component. Peak response rate was more resistant to change in the richer component during the VT and prefeeding tests, and change in peak rate was more sensitive to differential reinforcement than change in overall response rate. Changes in measures of performance on peak trials during the disruptor tests were partially consistent with predictions of the behavioral theory of timing. The results suggest that peak response rate provides a more sensitive index of resistance to change for fixedinterval schedules than does overall response rate and that reinforcement strengthens both peak responding and temporal control.
\end{abstract}

Nevin and colleagues proposed that response strength can be measured as the resistance to change of behavior when a disruptor, such as prefeeding, response-independent food, or extinction, is applied. In a typical experiment, Nevin (1974) arranged two variable-interval (VI) schedules for pigeons in an operant chamber. The schedules differed in terms of their reinforcement rate (rich or lean) and were signaled by distinctive stimuli (i.e., a two-component multiple schedule). After baseline training, free food was presented independent of responding between the components. Nevin (1974) found that response rate during the rich schedule decreased less, relative to baseline, than did response rate during the lean schedule. These results and others have led to the development of behavioral momentum theory, which assumes that resistance to change and response rate are analogous to the mass and velocity of a moving object in Newtonian mechanics. According to this theory, resistance to change is a measure of response strength, or behavioral mass, which is positively related to the rate, im-

This research was supported by NSF Grant IBN-9507584 to the University of New Hampshire (J.A.N., principal investigator). We thank Greg Fetterman and the anonymous reviewers for helpful comments and Melissa Bedell for assistance in conducting the experiments. Correspondence concerning this article should be addressed to $\mathrm{R}$. $\mathrm{C}$. Grace, University of Canterbury, Department of Psychology, Private Bag 4800, Christchurch, New Zealand, or to J. A. Nevin, RR \#2 Box 162, Vinehard Haven, MA 02568 (e-mail: r.grace@psyc.canterbury.ac.nz or tnevin@worldnet.att.net). mediacy, or magnitude of reinforcement associated with the response (Nevin, Mandell, \& Atak, 1983). Moreover, the positive correlation between behavioral mass and value, as measured by preference in concurrent chains, suggests that mass and value may represent a unitary construct determined by reinforcement history (Grace \& Nevin, 1997; Nevin \& Grace, 2000).

Resistance to change has usually been measured as the $\log$ proportion of the baseline response rate, $\log \left(B_{x} / B_{o}\right)$, where $B_{x}$ is the response rate during the disruptor test and $B_{o}$ is the baseline response rate (logarithms are taken to render equal ratios as equal intervals; for discussions of measurement issues, see Grace \& Nevin, 1997, and Nevin, Smith, \& Roberts, 1987). Relative resistance to change is then quantified as the difference in log proportion of baseline, $\log \left(B_{x 1} / B_{o 1}\right)-\log \left(B_{x 2} / B_{o 2}\right)$ (Grace \& Nevin, 1997). This approach seems appropriate for VI schedules, which typically sustain steady responding; indeed, most research on resistance to change has used VI schedules (cf. Cohen, Riley, \& Weigle, 1993). However, it could raise problems for fixed-interval (FI) schedules, in which responding shows temporal control: Response rates are low early in the interval and increase as the time of reinforcement approaches (Ferster \& Skinner, 1957). Examining only the change in overall response rate may fail to capture orderly changes in behavior that depend on the temporal pattern of responding in FI schedules.

One promising way to investigate whether resistance to change may depend on the temporal patterning of behavior is to use a version of the peak procedure. The peak 
procedure is a discrete-trial FI schedule that is popular in research on timing (Catania, 1970; Roberts, 1981). There are two types of trials in the peak procedure, food trials and peak (or no-food) trials, both of which begin with the onset of a stimulus. On food trials, the first response after a fixed delay has elapsed is reinforced with food. On peak trials, the stimulus remains present until well after the fixed delay, and the trial ends independent of responding. After sufficient baseline training, a typical pattern emerges when data from peak trials are aggregated: Responding begins at a low rate early in the trial, reaches a maximum or peak at about the time of reinforcement delivery on food trials, and declines afterward. In effect, the peak procedure measures both the increasing and the decreasing limbs of the FI response rate function.

Most studies using the peak procedure employ a single stimulus and FI schedule. However, a multiple peak procedure with two FI schedules can be arranged and would be analogous to the multiple VI schedules used in Nevin's prior work (see Roberts, 1981). Thus, the present experiments used a multiple peak procedure to explore the effects of three disruptors - response-independent food delivered between the components according to a variable-time (VT) schedule, prefeeding, and extinction-on the temporal pattern of pigeons' responding on peak trials. Differential reinforcement in the two components was established in three ways, by arranging (1) unequal magnitudes of reinforcement (Experiment 1), (2) FI schedule values (Experiment 2), and (3) probabilities of reinforcement on food trials (Experiment 3). For each disruptor test, we examined changes in various measures of behavior on peak trials. In particular, we asked whether changes in peak response rate would provide a better measure of behavioral mass (in terms of being more sensitive to differences in reinforcement) than would change in overall response rate. The general prediction from behavioral momentum theory is that responding in the richer component will be more resistant to change than responding in the leaner component will be. Resistance to extinction may not conform to this pattern, however, because the omission of reinforcers will have a larger impact, via generalization decrement, on performance trained with a higher rate of reinforcement (Nevin \& Grace, 1999).

A secondary goal of our research was to assess whether effects of the disruptors on peak trial responding were consistent with current theories of timing. The predominant theories of animal timing are Gibbon's (1977) scalar expectancy theory (SET) and Killeen and Fetterman's $(1988,1993)$ behavioral theory of timing (BeT; see also Machado, 1997). However, only the BeT makes clear predictions about disruptor effects in the present experiments.

According to the BeT, timing is mediated by a sequence of transitions through various behavioral states. These transitions are controlled by pulses emitted from a Poisson pacemaker. Consequently, the probability dur- ing a peak trial that a subject is in the state associated with responding is given by a gamma distribution (Beam, Killeen, Bizo, \& Fetterman, 1998), which is characterized by a mean and a standard deviation:

$$
\mu=\tau(n+1)
$$

and

$$
\sigma=\tau \sqrt{n+1},
$$

where $\tau$ is the period of the pacemaker and $n$ is the number of the behavioral state associated with reinforcement on food trials. Although the BeT assumes that the speed of the pacemaker is proportional to reinforcement rate, data suggest that the relationship is linear with a nonzero intercept (e.g., Fetterman \& Killeen, 1995). Because the disruptors used here may be construed as changes in overall reinforcement rate, BeT makes several predictions for peak-trial responding in the present experiments.

Addition of response-independent food should increase the speed of the pacemaker (i.e., reduce $\tau$ ), leading to decreases in peak location and variability. The decrease in peak location should be only temporary, since the birds will recalibrate their performance by changing $n$ to keep the mean at the time of reinforcement (Fetterman \& Killeen, 1991). However, the decrease in variability will be durable, because recalibration will not restore the original standard deviation (since it depends on the square root of $n$ ). Consistent with the BeT, Fetterman and Killeen's (1995) results suggest that the mean, but not the standard deviation, recalibrated when the probability of reinforcement was changed in a three-choice categorical scaling procedure.

Extinction is a decrease in reinforcement rate and thus should reduce the speed of the pacemaker (i.e., increase $\tau$ ), producing durable increases in peak location and variability. There is no opportunity for recalibration to occur. Killeen, Hall, and Bizo (1999) found that, as predicted, extinction increased estimates of $\tau$ in a psychophysical choice procedure.

Although prefeeding does not change the reinforcement rate in the session itself, the overall access to food for the pigeons is increased. If so, it is reasonable to expect that prefeeding will be similar to response-independent food and will produce durable decreases in the variability, but not in the location, of the response distributions. Thus, for the present experiments, the BeT predicts that responseindependent food and prefeeding should produce temporary decreases in peak location and lasting decreases in the variability of peak-trial responding, whereas extinction should increase both peak location and variability. Note that apart from extinction, the disruptors are "off baseline" (see Morgan, Killeen, \& Fetterman, 1993).

Theories of timing predict no differences between the rich and the lean components in baseline, with the exception of Experiment 2, in which peak location should track the FI schedule and where variability should be 
proportional to the mean (i.e., Weber's law). According to the SET, pigeons begin responding on an FI schedule when the expectancy to reinforcement, which increases as the trial elapses, exceeds the background or overall expectancy value in a ratio comparison. Because expectancy is defined as the product of reinforcement immediacy and magnitude, the SET predicts that peak location and variability should be the same for both components in Experiments 1 and 3 (as differential magnitude or probability cancels in the expectancy ratio). Beam et al. (1998) found that for the BeT, pacemaker speed can come under stimulus control and be greater in the richer component. However, to the extent that pacemaker speed differs, the pigeons will learn different criterial numbers of behavioral states, with the net result that peak-trial responding should be equivalent in the two components in Experiments 1 and 3 .

Thus, there were three major questions posed in each experiment: (1) whether there were baseline differences in temporal control between the rich and the lean components, (2) what the effects were, if any, of the disruptors on temporal control, and (3) whether there were systematic differences between the rich and the lean components in terms of how the pattern or rate of responses on peak trials was disrupted.

\section{GENERAL METHOD}

\section{Apparatus}

Four standard operant chambers, $35 \mathrm{~cm}$ deep $\times 35 \mathrm{~cm}$ wide $\times$ $35 \mathrm{~cm}$ high, were used. In each chamber, there were three response keys, located $26 \mathrm{~cm}$ above the floor and arranged in a row, a houselight $7 \mathrm{~cm}$ above the center key, and a grain magazine with a $6 \times$ $5 \mathrm{~cm}$ aperture $13 \mathrm{~cm}$ below the center key. The magazine was illuminated when wheat was made available. A force of approximately $0.10 \mathrm{~N}$ was necessary to operate each key and to produce an audible feedback click. Chambers were enclosed in a sound-attenuating box, and ventilation and white noise were provided by an attached fan. Experimental events were controlled and data were recorded with a microcomputer located in an adjacent room.

\section{Procedure}

All the experiments used a modified peak procedure (Roberts, 1981 ) in which subjects were exposed in baseline training to two FI schedules that differed in terms of reinforcement magnitude, immediacy, or probability. Sessions contained 72 trials. At the start of a trial, the houselight was turned on, and the center key was illuminated either red or green. The center key color was determined randomly, with the restriction that there were 4 of each color in every 8 trials. For each color, there were two types of trials: regular trials, on which reinforcement might be available, and peak trials, on which no reinforcement was available. In every 4 trials of each color, there were 3 regular trials and 1 peak trial. Thus, sessions consisted of 36 red and 36 green trials, with 27 regular and 9 peak trials of each color. There was an FI schedule associated with each color that made reinforcement available after a fixed delay, with a specified magnitude (i.e., duration of access to grain) and probability.

On regular trials, the first response after the FI schedule value had elapsed terminated the trial, provided that it occurred within $5 \mathrm{sec}$. Whether or not that response was reinforced was determined probabilistically, with the number of reinforced regular trials (out of 27) per session being specified for each schedule (only in Experi- ment 3 was the number ever less than 27). During reinforcement, the keylight and houselight were turned off, and the grain magazine was raised and illuminated for a specified duration. If the response was not reinforced, the keylight and houselight were turned off, and the trial ended. In addition, if the response did not occur within $5 \mathrm{sec}$ of the FI schedule's having elapsed, reinforcement was canceled, and the trial ended.

Peak trials lasted for three times the FI schedule value and ended independent of responding. No reinforcement was ever available on peak trials, and responses were recorded in 1-sec bins. All the trials were followed by an intertrial interval (ITI) of $20-\mathrm{sec}$ duration, during which all the lights in the chamber were extinguished.

After baseline training in each condition, three types of resistanceto-change tests were conducted, in which responding on regular and peak trials was disrupted. The disruptors were response-independent food delivered during the ITI (VT food), homecage prefeeding, and extinction.

Variable-time food. In VT food test sessions, grain was made available during the ITI, independent of responding, according to a VT 7.5-sec schedule. The intervals for the VT schedule were defined as an exponential progression (Fleshler \& Hoffman, 1962) and were sampled without replacement. VT food resistance tests consisted of at least five sessions, during which food was presented during the ITI. The subjects were then returned to $85 \%$ of their ad-lib weights and were given additional baseline training prior to the prefeeding test.

Prefeeding. In the first two sessions, the subjects were prefed 20 and $40 \mathrm{~g}$ of pigeon chow in their home cages one-half hour prior to session time. For the third and subsequent sessions, the amount prefed was $60 \mathrm{~g}$. All the prefeeding tests were conducted for at least five consecutive sessions. After completing the prefeeding sessions, the subjects were returned to $85 \%$ of their ad-lib weights and were given additional baseline training prior to the extinction test.

Extinction. During extinction sessions, no reinforcement was available. On all the regular trials, the first response that met the FI schedule criterion terminated the trial. As in baseline, regular trials ended independent of responding if the subject failed to make a response within $5 \mathrm{sec}$ of the FI schedule's having elapsed. Peak trials remained as in baseline. Extinction continued for at least five sessions.

To provide comparability across disruptors and experiments and for consistency with prior research, data from the first five sessions in each disruptor test were compared with the immediately preceding five baseline sessions. In some cases, additional test sessions were given to explore the effects of long-term disruptors on timing performance. The resistance tests were conducted in the same order in each experiment, but because the results from each test are compared with the prior baseline, this should not raise problems.

Measures of peak-trial performance. We used nonparametric measures of peak location and variability to assess the predictions of behavioral momentum theory and the BeT. The most important reason for this approach is that reliable estimates of the median and interquartile range may be obtained from single-session data, allowing for a more fine-grained analysis than when data must be pooled across sessions (see Grace \& Nevin, 1999). But to ensure that our nonparametric measures were robust, we also fitted normal densities to the response distributions and assessed the comparability of the mean, standard deviation, and recovered height of the distributions to the nonparametrics.

The location of the response peak was obtained by using the trimmed median method of Cheng and Roberts (1991). A cumulative response distribution was generated, and the bin that contained the median response was located. The median time was then calculated by linear interpolation. Another cumulative distribution was generated after excluding all responses that occurred after twice that median. A new median was then calculated, and the process was iterated until successive medians stopped changing. The final 
median was taken as an estimate of the peak time. To provide a measure of variability, the interquartile range was computed as the difference between the time values for the $75 \%$ and the $25 \%$ percentile responses, each calculated through linear interpolation. An index of temporal sensitivity analogous to the coefficient of variation is provided by the interquartile range divided by the median (iqr/m). Finally, the peak response rate was calculated as the response rate at the peak time, again using linear interpolation with the two bins on either side of the median.

Statistical tests used a .05 significance level, all $t$ tests were twotailed, and post hoc comparisons used the Bonferroni method.

\section{EXPERIMENT 1}

In Experiment 1, we manipulated reinforcement magnitude. Responses in one component produced 1.5-sec of access to grain (small), whereas responses in the other component produced $4.5 \mathrm{sec}$ of access to grain (large). Prior research with multiple schedules with VI components has found that responding in the component that provides the relatively greater reinforcement magnitude is more resistant to the disruptive effects of VT food, prefeeding, and extinction (Harper, 1996; Harper \& McLean, 1992; Nevin, 1974; Nevin, Mandell, \& Yarensky, 1981; Shettleworth \& Nevin, 1965). Thus, an additional question posed by Experiment 1 is whether this result would generalize to FI schedules.

\section{Method}

Subjects. The subjects were 4 White Carneaux pigeons. They were maintained at $85 \%$ ad-lib weight, plus or minus $15 \mathrm{~g}$, by postsession feedings and were housed in a vivarium with a 12:12-h light:dark cycle (lights on at 0700). All the birds had experience with multiple schedules and concurrent chains but were naive as to the peak procedure.

Procedure. Because the subjects were experienced, baseline training began immediately on a multiple peak procedure. In the large-magnitude component, responses were reinforced with $4.5-\mathrm{sec}$ access to grain according to an FI 20 -sec schedule; responses in the small-magnitude component produced 1.5-sec access to grain according to the same schedule. Beinforcement was available on $100 \%$ of the regular trials in each component. For 2 birds, the center key was lighted red during the small-magnitude component and green during the large-magnitude component; this assignment was reversed for the other birds. With few exceptions, sessions were conducted 7 days per week at approximately the same time.

Baseline training consisted of 28 sessions and was followed by the VT food test. During this test, which lasted for 20 sessions, grain magazine presentations (1.67-sec duration) were delivered during the ITI according to a VT 7.5-sec schedule. The subjects then completed 11 baseline sessions prior to the prefeeding test, which lasted for 13 sessions. The subjects were returned to baseline for 12 additional sessions before the extinction test, which lasted for 10 sessions.

\section{Results}

Figure 1 shows response rate as a function of elapsed time during peak trials over the last five sessions of baseline and the first five disruptor sessions, for both the small- (1.5-sec) and large- $(4.5-\mathrm{sec})$ magnitude components. The data are averaged across subjects. The over- all pattern of responding in baseline is typical of peak procedure performances: a steady increase in response rate rising to a maximum at approximately the FI schedule value, followed by a decrease. The baseline data in Figure 1 were assessed by a series of repeated measures analyses of variance (ANOVAs), with disruptor test (i.e., the baseline determination prior to each test) and magnitude as factors. For all subjects, peak location as measured by the median was significantly less in the largemagnitude component $(M=20.47)$ than in the smallmagnitude component $[M=22.29 ; F(1,3)=12.13]$. Analysis of timing sensitivity $(\mathrm{iqr} / \mathrm{m})$ found an interaction between disruptor test and magnitude $[F(2,6)=$ $7.42]$, and post hoc comparisons showed that $i q r / m$ was lower in the small-magnitude component in baseline preceding each disruptor test (i.e., greater relative sensitivity). There were no significant differences in variability (as measured by interquartile range) or peak response rate.

Did the disruptors have systematic effects on measures of timing performance? Figure 1 suggests that responding decreased when the disruptors were applied and the reduction in responding was relatively greater in the small-magnitude component. For a more precise assessment of disruptor effects, we analyzed single-session data. Figure 2 shows four measures of performance on peak trials - median, interquartile range, peak response rate, and iqr $/ m$-over the last five sessions of baseline and the first five sessions of each disruptor test, averaged across subjects. The results for median and interquartile range are shown in the left panels of Figure 2; peak response rates are shown in the center panels of Figure 2. The data were entered into repeated measures ANOVAs for each dependent variable, with magnitude, session type (baseline/test), disruptor type, and session as factors. For the median, a significant effect was found for magnitude $[F(1,3)=95.88]$, and the magnitude $X$ session type interaction was significant $[F(1,3)=20.24]$. For interquartile range, the main effect of session type $[F(1,3)=10.03]$ and the magnitude $\times$ session type interaction $[F(1,3)=10.64]$ were significant. For peak response rate, the main effects of magnitude $[F(1,3)=$ $12.11]$ and session $[F(4,12)=4.37]$ and both the session type $\times$ disruptor type $[F(2,6)=8.25]$, and the magnitude $X$ disruptor type $X$ session $[F(4,12)=3.92]$ interactions were significant.

Post hoc comparisons carried out between the baseline and the disruptor test for each component found that the increases in the medians for the large-magnitude component in the VT test and the small-magnitude component in extinction were significant, as was the increase in interquartile range for the small-magnitude component during prefeeding. The increase in interquartile range for the small-magnitude component during extinction and for both components during the VT test approached significance. All the decreases in peak response rate were significant. Thus, Figure 2 indicates that the median and 


\section{Magnitude - $1.5 \mathrm{~s}$}

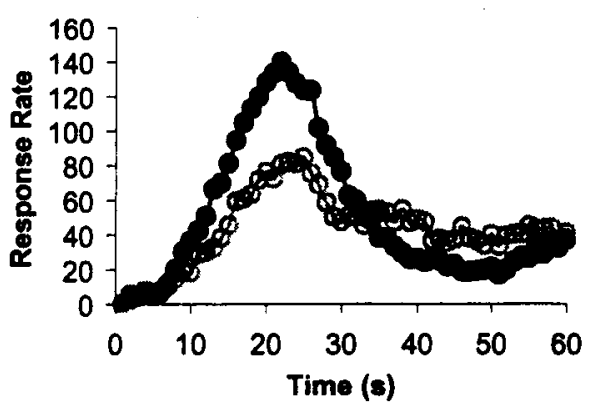

\section{Magnitude - $1.5 \mathrm{~s}$}

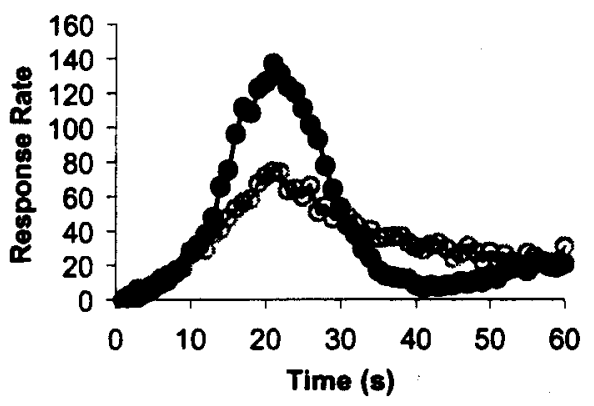

Magnitude - $1.5 \mathrm{~s}$

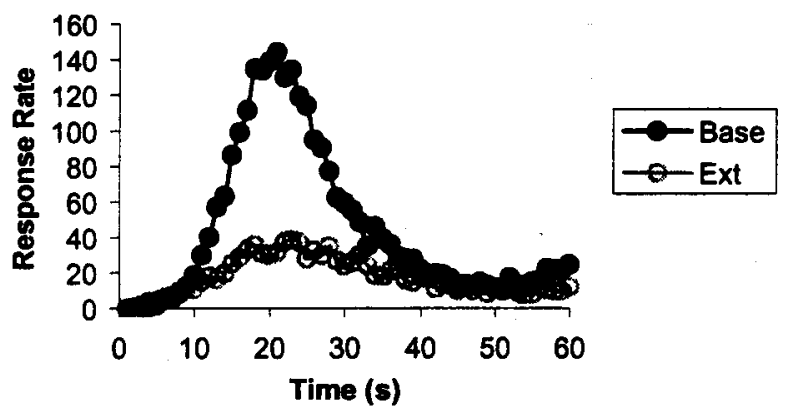

Magnitude $-4.5 \mathrm{~s}$

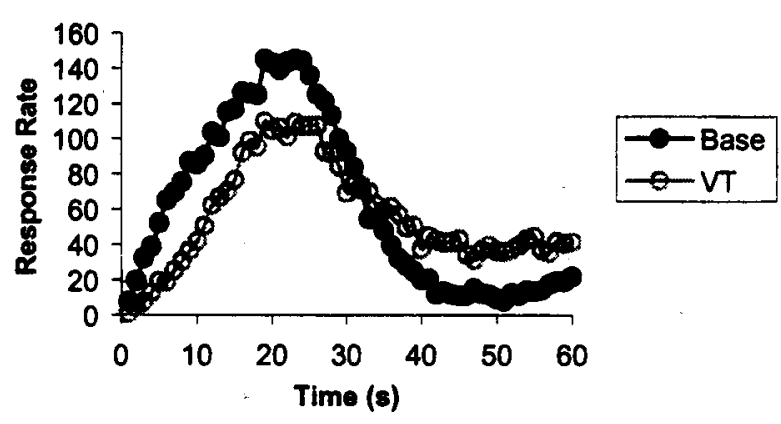

Magnitude $-4.5 \mathrm{~s}$

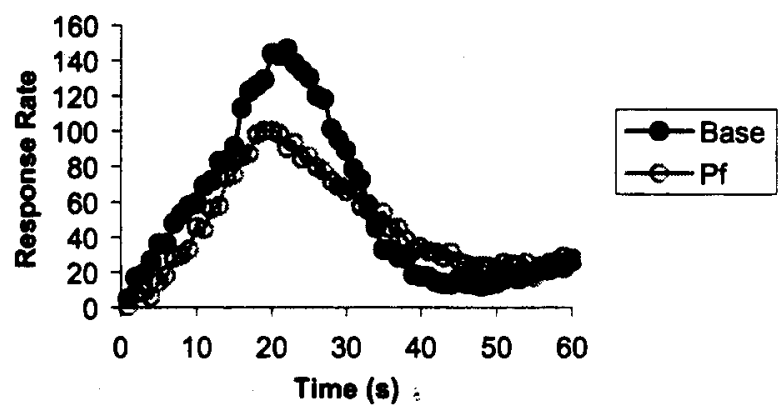

Magnitude - $4.5 \mathrm{~s}$

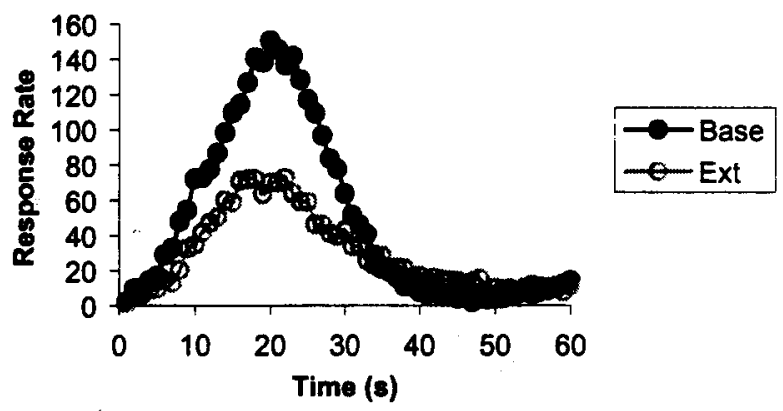

Figure 1. A comparison of peak-trial responding between baseline and the three resistance to change tests from Experiment 1 . In each panel, the filled circles represent responding on the five baseline sessions preceding a resistance test, and the unfilled circles show responding on the first five sessions of the resistance test. The data from the variable-time, prefeeding, and extinction tests are shown in the upper, middle, and lower panels, respectively. The results from the small-magnitude component (1.5 sec) are displayed in the left column; the results from the large-magnitude component $(4.5 \mathrm{sec})$ are shown in the right column. The data are averaged across subjects.

the interquartile range either increased or remained about the same, whereas peak response rate decreased, for all the disruptor tests. ${ }^{1}$

The average values of $i q r / m$ over the last five baseline and the first five disruptor test sessions are shown in the right panels of Figure 2 . The main effect of session type was significant $[F(1,3)=37.05]$, whereas the main ef- fect of disruptor type and the disruptor type $\times$ magnitude $\times$ session type interaction approached significance (both $p \mathrm{~s}=.06$ ). Post hoc comparisons carried out between the baseline and the disruptor test for each component found that increases in the small-magnitude component for the prefeeding and extinction tests reached significance. Thus, the data in Figure 2 indicate that 

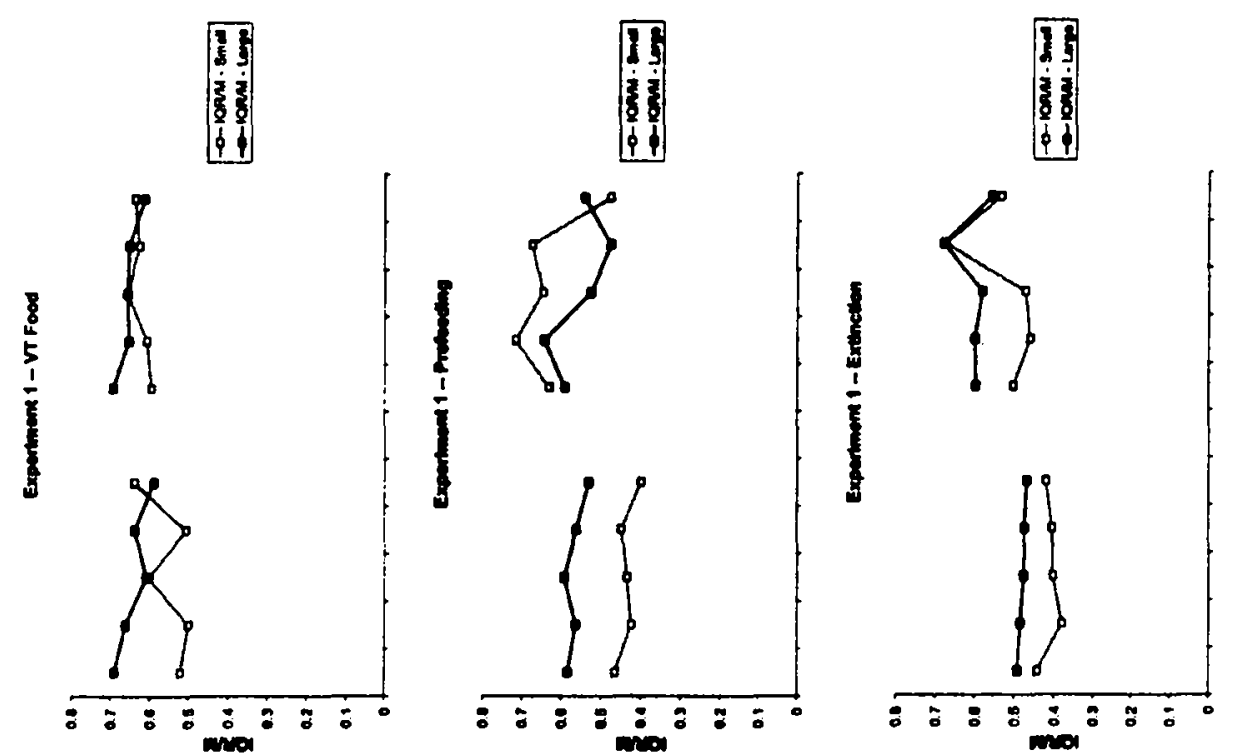

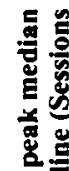
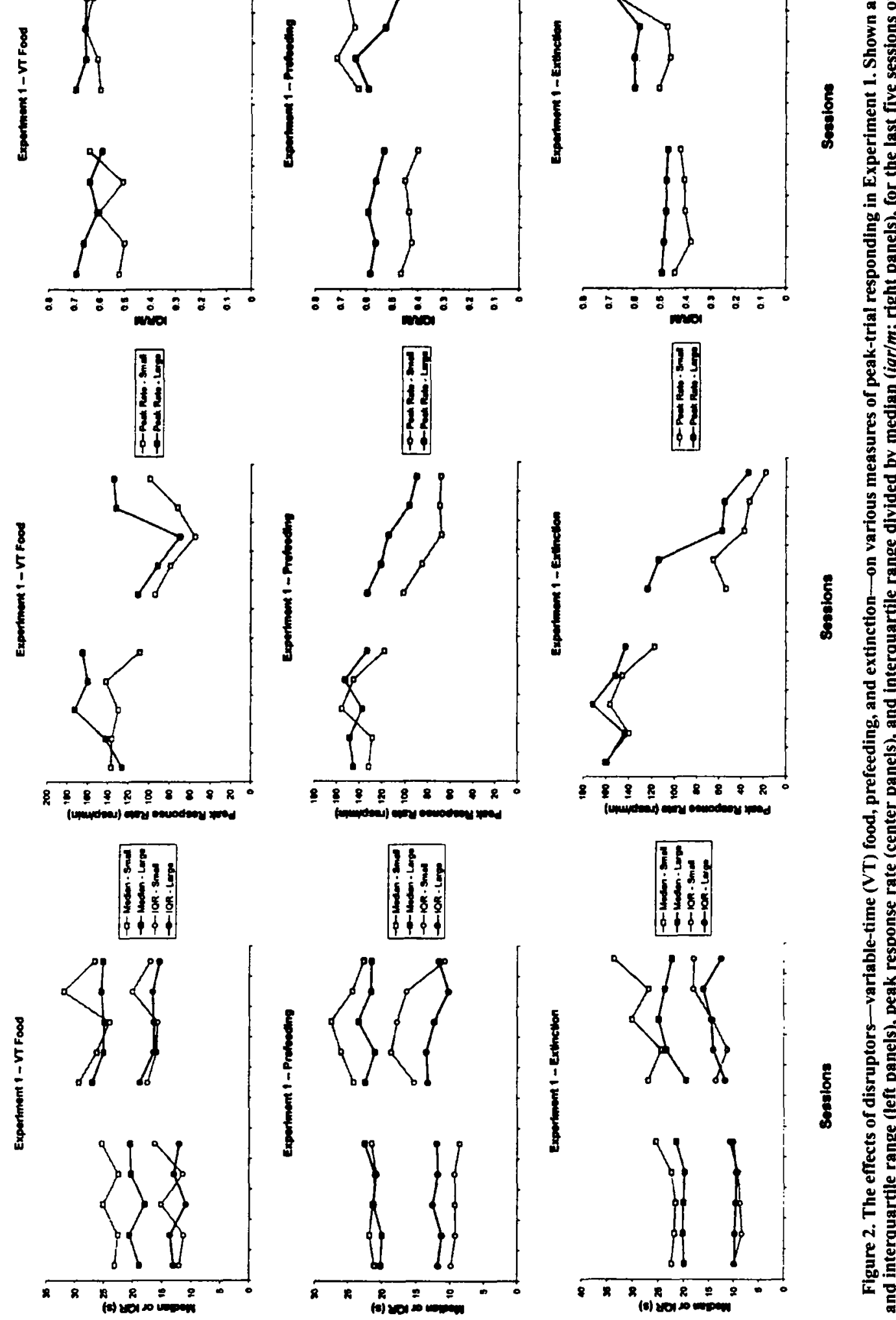

है

这

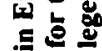

흠

믐

兔

든

妾

늠

串

焉

势

웄 흘

$\frac{8}{8}$

突

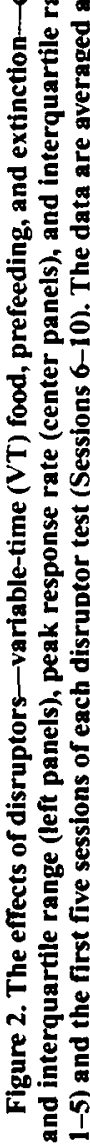


Table 1

Resistance to Change for Experiment 1, Measured as the Log Proportion of Baseline: Results for Overall Response Rate and Four Measures of Performance on Peak Trials

\begin{tabular}{|c|c|c|c|c|c|c|c|c|c|c|}
\hline \multirow[b]{3}{*}{ Disruptor Test } & \multicolumn{10}{|c|}{ Measure } \\
\hline & \multicolumn{2}{|c|}{ Overall Response Rate } & \multicolumn{2}{|c|}{ Median } & \multicolumn{2}{|c|}{$i q r$} & \multicolumn{2}{|c|}{ Peak Rate } & \multicolumn{2}{|c|}{$i q r / m$} \\
\hline & Average & $S E$ & Average & $S E$ & Average & $S E$ & $\overline{\text { Average }}$ & $S E$ & Average & $S E$ \\
\hline VT, small & -.19 & .15 & .09 & .04 & .16 & $.05^{*}$ & -.22 & .13 & .07 & $.04^{*}$ \\
\hline VT, large & -.13 & .09 & .10 & .03 & .09 & $.04^{*}$ & -.09 & .07 & -.01 & $.03 *$ \\
\hline Prefeeding, small & -.13 & .08 & .07 & .03 & .23 & $.06^{*}$ & -.31 & $.09^{*}$ & .16 & $.04^{*}$ \\
\hline Prefeeding, large & -.14 & .06 & .02 & .04 & .04 & $.03^{*}$ & -.10 & $.05^{*}$ & .01 & $.00^{*}$ \\
\hline Extinction, small & -.43 & $.10^{*}$ & .05 & .05 & .19 & .06 & -.60 & $.07^{*}$ & .14 & .03 \\
\hline Extinction, large & -.30 & $.10^{*}$ & .04 & .05 & .15 & .07 & -.40 & $.10^{*}$ & .11 & .04 \\
\hline
\end{tabular}

Note-iqr, interquartile range; $i q r / m$, interquartile range divided by the median; VT, variable time. Positive values indicate increases, relative to baseline; negative values indicate decreases. The data are averaged across subjects. Pairs of values followed by asterisks were significantly different $(p<.05)$ in a planned comparison.

$i q r / m$ either increased or remained unchanged during each disruptor test.

Behavioral momentum theory predicts that responding in the large-magnitude component will be more resistant to change than responding in the small-magnitude component will be. We computed resistance to change, using the traditional measure, $\log$ proportion of overall response rate (on both regular and peak trials). In addition, we explored measures of resistance to change derived from performance on peak trials, including the log proportion of baseline median, interquartile range, peak response rate, and $i q r / m$. All the measures were computed on the aggregated (five sessions) baseline and disruptor peak trial distributions. The average results are shown in Table 1. Individual data were entered into a series of repeated measures ANOVAs, with type of disruptor and magnitude as factors. Planned comparisons were then used to determine whether resistance to change systematically differed between the small- and the largemagnitude components for each disruptor test. For resistance to change as measured by changes in overall response rate, the main effect of disruptor type was significant $[F(2,6)=14.33]$. Planned comparisons found that response rate decreased more, relative to baseline, in the small-magnitude component during extinction. For resistance to change as measured by change in peak median, there were no significant effects. With interquartile range, the effect of magnitude was significant $[F(1,3)=$ 40.89]. Planned comparisons showed that the interquartile range increased more, relative to baseline, in the small-magnitude component during prefeeding. For peak response rate, the main effects of disruptor type $[F(2,6)=$ 13.35] and magnitude $[F(1,3)=10.02]$ were significant. Planned comparisons found that peak response rate decreased less, relative to baseline, in the rich component for the prefeeding and extinction tests. For $i q r / m$, the main effect of magnitude was significant $[F(1,3)=$ $33.61]$, and planned comparisons found that $\mathrm{iqr} / \mathrm{m}$ increased more, relative to baseline, in the small component during both the VT and the prefeeding tests. Note that the smaller the value of $i q r / m$, the greater temporal sensitivity, so larger increases correspond to weaker temporal control.

Because the VT food, prefeeding, and extinction tests lasted for 20,13 , and 10 sessions, respectively, we explored whether timing performance changed systematically over the course of each test. There were no changes in median or interquartile range during the course of prefeeding or extinction. The VT results are displayed in Figure 3, which shows the log proportion of baseline for peak median, interquartile range, $i q r / m$, and peak response rate for successive 5-session blocks of the test. Both the median and the interquartile range increased over the baseline during the first block and decreased thereafter $[F(3,9)=9.16$ and $F(3,9)=17.58]$. Similarly, $i q r / m$ decreased over the course of the VT test $[F(3,9)=$ 26.25], and greater decreases were obtained in the largemagnitude component $[F(1,3)=23.64]$. Peak response rate did not decrease over the course of the VT test.

\section{Discussion}

Behavioral momentum theory predicts that resistance to change (typically measured as the log proportion of baseline response rate) should be greater in the largemagnitude component. The results were mixed. Overall, response rate decreased less in the large-magnitude component, relative to baseline, during the VT and extinction tests, but the differences were significant only for extinction. However, when resistance to change was measured as the log proportion of peak response rate, the results were more consistent with theory: Peak responding decreased less in the large-magnitude component for all disruptor tests, and differences reached significance for both prefeeding and extinction. This finding provides some tentative support for our hypothesis that peak response rate provides a more sensitive measure than does change in overall response rate. In addition, relative temporal sensitivity (measured as $\mathrm{i} g \mathrm{r} / \mathrm{m}$ ) revealed similar effects of reinforcement magnitude: $i q r / m$ increased more, relative to baseline, in the small-magnitude component 
Median

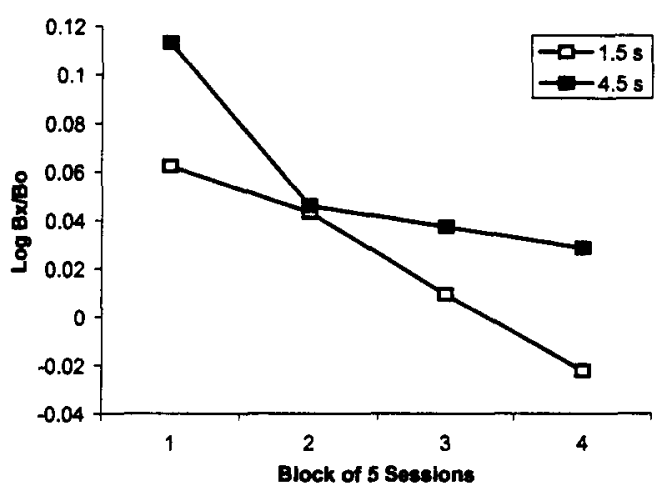

Interquartlle Range

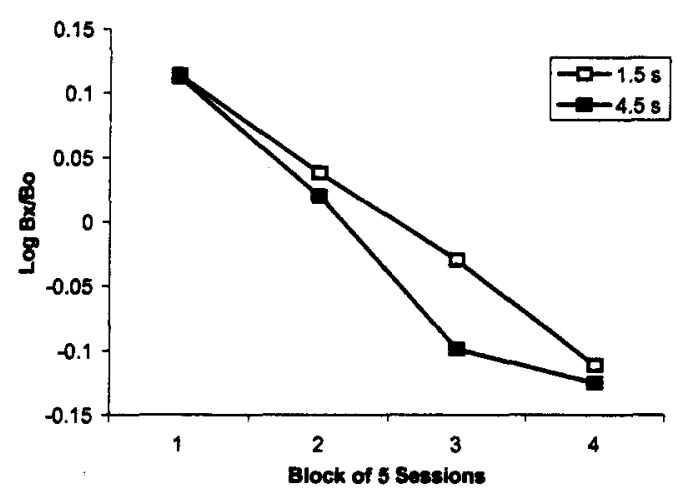

iqr $/ \mathbf{m}$

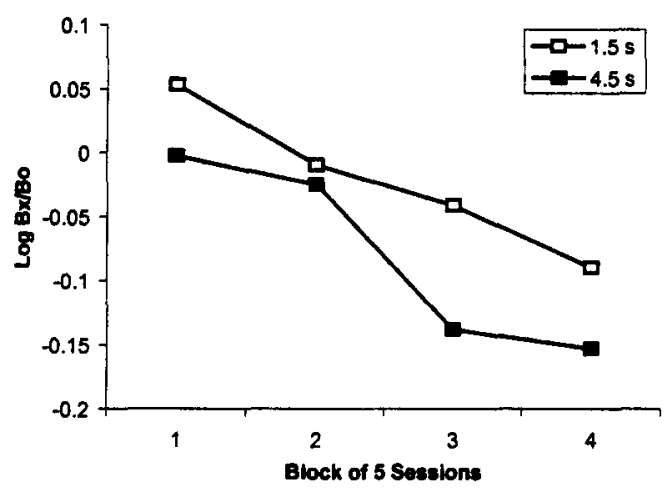

Peak Response Rate

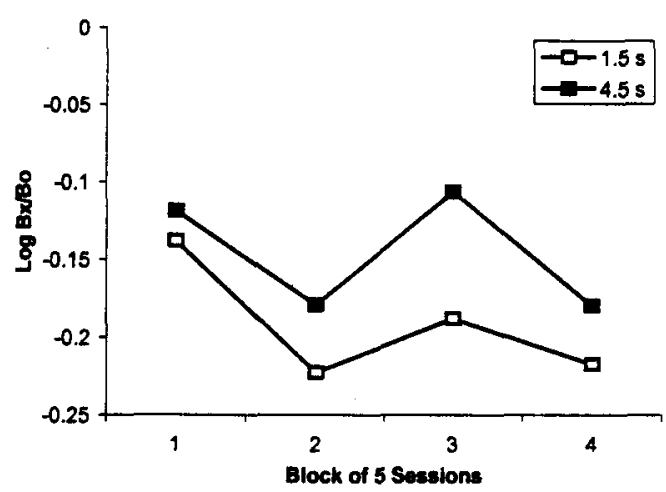

Figure 3. This figure shows how the effects of variable-time (VT) food on measures of peak-trial responding (median peak time, interquartile range, interquartile range divided by median, and peak response rate, measured as $\log$ proportion of baseline) changed over the course of $20 \mathrm{VT}$ test sessions. The results for the large-magnitude component are shown by filled symbols, and those for the small-magnitude component by unfilled symbols. The data are averaged across subjects.

in all the disruptor tests, and the differences during VT and prefeeding reached significance.

The effects of disruptors on peak-trial responding provided partial support for the predictions of the BeT. During extinction, peak location and variability increased during the small-magnitude component. According to the BeT, pacemaker speed should decrease in extinction, owing to the downward shift in reinforcement rate. The peak location and variability should increase (by Equations 1 and 2), and this is just what we observed. However, the BeT also predicts that the VT test and prefeeding should decrease variability but leave peak location unchanged. This prediction was not supported, at least in the initial sessions of the disruptor tests. On average, the interquartile range increased in the small-magnitude component in all tests and in the large-magnitude component during the VT test. Moreover, the medians increased, rather than remaining unchanged, in the largemagnitude component during VT and in the smallmagnitude component during extinction.
However, the increases in median and interquartile range during VT were transient. An analysis of the data over the full 20 sessions of the VT test showed that the median returned to baseline levels and that the interquartile range dropped below baseline. Thus, by the end of the VT test, the data fully supported the BeT's predictions. Yet, because the temporary increases must have been mediated by changes in pacemaker speed (which presumably would be in the wrong direction), it is unclear whether the entire pattern of results can be explained by the BeT.

Theories of timing predicted no difference in baseline peak-trial responding. However, peak location occurred earlier in the large-magnitude component. This finding conflicts with the results of MacEwen and Killeen (1991), who found no effect of reinforcement magnitude on peak location (and inferred pacemaker speed, $\tau$ ). One possible reason for the difference between the studies is that MacEwen and Killeen used a single-component procedure and their results were based on manipulating rein- 
forcement magnitude across conditions, rather than within conditions, as in the present experiment (see also Beam et al., 1998).

\section{EXPERIMENT 2}

In Experiment 2, we investigated the effects of reinforcement rate (or immediacy) on timing and resistance to change. Responses were reinforced according to an FI 10 -sec schedule in one component and an FI 30-sec schedule in the other. When VI schedules with different reinforcement rates are arranged in multiple schedules, the typical result is that the component with the richer reinforcement rate is more resistant to change (Nevin et al., 1983). Analogous results were obtained with FI schedules by Cohen et al. (1993). Thus, we expected that responding during peak trials for the FI 10-sec schedule would change less, relative to baseline, than peak-trial responding for the FI 30-sec schedule. Theories of timing predict that performances on the FI 10-sec and FI 30sec components should satisfy Weber's law; that is, relative sensitivity (as indexed by iqr $/ \mathrm{m}$ ) should be the same for both components in baseline.

\section{Method}

Subjects. The subjects from Experiment 1 served. Training in Experiment 2 began immediately after the conclusion of Experiment 1 .

Procedure. The same multiple peak procedure was used, except that different FI schedule values were used with equal reinforcement magnitudes. In the rich component, responses were reinforced according to an FI 10-sec schedule; an FI 30-sec schedule was used in the lean component. Reinforcement consisted of $3 \mathrm{sec}$ of access to grain and was available on $100 \%$ of the regular trials in each component. Peak trials lasted $3 \times$ the schedule values (i.e., $30 \mathrm{sec}$ for FI $10 \mathrm{sec} ; 90 \mathrm{sec}$ for FI $30 \mathrm{sec}$ ). For 2 birds, the center key was lighted red during the lean component and green during the rich component; this assignment was reversed for the other birds. Sessions were generally conducted 7 days per week at approximately the same time.

Baseline training consisted of 28 sessions and was followed by the VT food test. During this test, which lasted 5 sessions, grain magazine presentations (1.67 $\mathrm{sec}$ duration) were delivered during the ITI according to a VT 7.5-sec schedule. The subjects then completed 13 baseline sessions prior to the prefeeding test, which lasted for 5 sessions. The subjects were returned to baseline for 24 additional sessions, followed by 5 sessions of extinction.

\section{Results}

Figure 4 shows response rate on peak trials aggregated over both the last five sessions of baseline and the first five sessions of each disruptor test and averaged across subjects. Pigeons discriminated the two intervals and timed them more or less accurately. The average baseline peak medians in Figure 4 were $34.36 \mathrm{sec}$ (FI 30) and $8.82 \mathrm{sec}$ (FI 10). For all the subjects, the median as a proportion of the schedule value was less for FI 10 than for FI $30[F(1,3)=10.82]$. This is consistent with the results of Experiment 1 in that the peak occurred earlier, relative to the schedule value in the richer component. Peak response rate was reliably greater for FI $10[F(1,3)=$
11.10]. In addition, there was a main effect of disruptor test on (baseline) peak response rate $[F(2,6)=12.65]$. Post hoc comparisons demonstrated that the baseline peak rate preceding extinction was greater than the baseline preceding either the prefeeding or the VT tests.

Figure 5 shows the peak median and interquartile range (left panels) and the peak response rate (center panels) from individual sessions during baseline and each disruptor test, averaged across subjects. Individual data from Figure 5 were entered into repeated measures ANOVAs, with disruptor type, component type (FI $10 \mathrm{sec}$ or FI $30 \mathrm{sec}$ ), session type (baseline or test), and session as factors. Although both the median and the interquartile range were greater in the FI 30 -sec component $[F \mathrm{~s}(1,3)=$ 354.94 and 30.88], there were no significant changes in these measures between baseline and test. For peak response rate, there were significant effects of component type $[F(1,3)=12.62]$ and session $[F(4,12)=9.45]$. Post hoc comparisons found that peak response rate decreased in both components during the prefeeding and extinction tests. Thus, peak response rates generally decreased during disruption, consistent with Experiment 1, although the median and interquartile range did not change systematically. Results for temporal sensitivity (iqr/m) are shown in the right panels of Figure 5. The disruptor type $\times$ component type interaction was significant $[F(2,6)=$ 8.29 ], and post hoc tests showed that $i q r / m$ was greater in the FI 10-sec component during baseline and test for VT and extinction. This result is also consistent with Experiment 1 , because it suggests that timing sensitivity was greater in the relatively leaner component (i.e., FI $30 \mathrm{sec})$.

Resistance to change, measured as log proportion of baseline, was assessed using overall response rate as well as the measures derived from peak trials (median, interquartile range, peak response rate, and $i q r / m$ ). All the measures were computed on the aggregated (five sessions) baseline and disruptor peak-trial distributions. Average values of log proportion of baseline for all measures are listed in Table 2 . Individual data were analyzed with a series of repeated measures ANOVAs, with type of disruptor and magnitude as factors and with planned comparisons to determine whether resistance to change systematically differed between the FI 30-sec and the FI 10 -sec components. For the traditional measure of resistance to change, overall response rate, there were no significant effects. With relative change in peak response rate as the measure of resistance to change, the effect of disruptor type was significant $[F(2,6)=5.13]$, and for all 4 subjects, peak response rate decreased less, relative to baseline, in the FI 10-sec component during prefeeding and in the FI 30 -sec component during extinction. There were no significant effects with resistance to change measured as log proportion of peak median or iqr $/ m$. With interquartile range, the component type $\times$ disruptor type interaction was significant $[F(2,6)=11.09]$, and comparisons showed that the interquartile range increased more, relative to baseline, in the FI 10-sec com- 

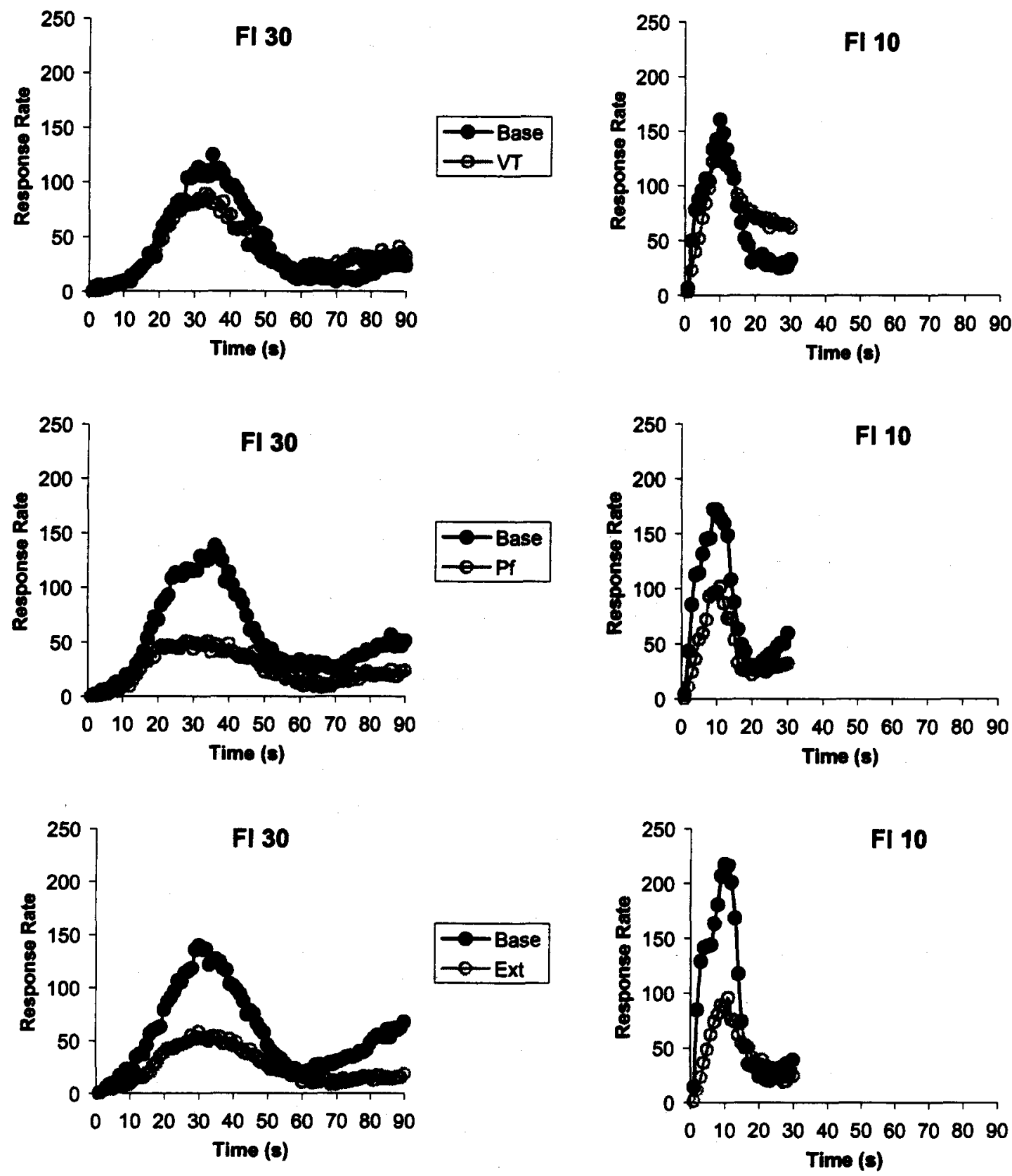

Figure 4. A comparison of peak-trial responding between baseline and the three resistance-to-change tests from Experiment 2 . In each panel, the filled circles represent responding on the five baseline sessions preceding a resistance test, and the unfilled circles show responding on the first five sessions of the resistance test. The data from the variable-time (VT), prefeeding, and extinction tests are shown in the upper, middle, and lower panels, respectively. Results from the FI 30-sec component are displayed in the left column; results from the FI 10-sec component are displayed in the right column. The data are averaged across subjects.

ponent during the VT and extinction tests, but more in the FI 30-sec component during prefeeding.

\section{Discussion}

Behavioral momentum theory predicts that responding should be more resistant to change in the FI 10-sec component. But as Table 2 shows, regardless of whether resistance to change was measured in terms of overall or peak response rate, there were no statistically significant differences on any of the disruptor tests. However, differences between components were more pronounced when peak response rates were used: For all the subjects, responding was more resistant to change in the FI 10-sec component during prefeeding, but more resistant in the 

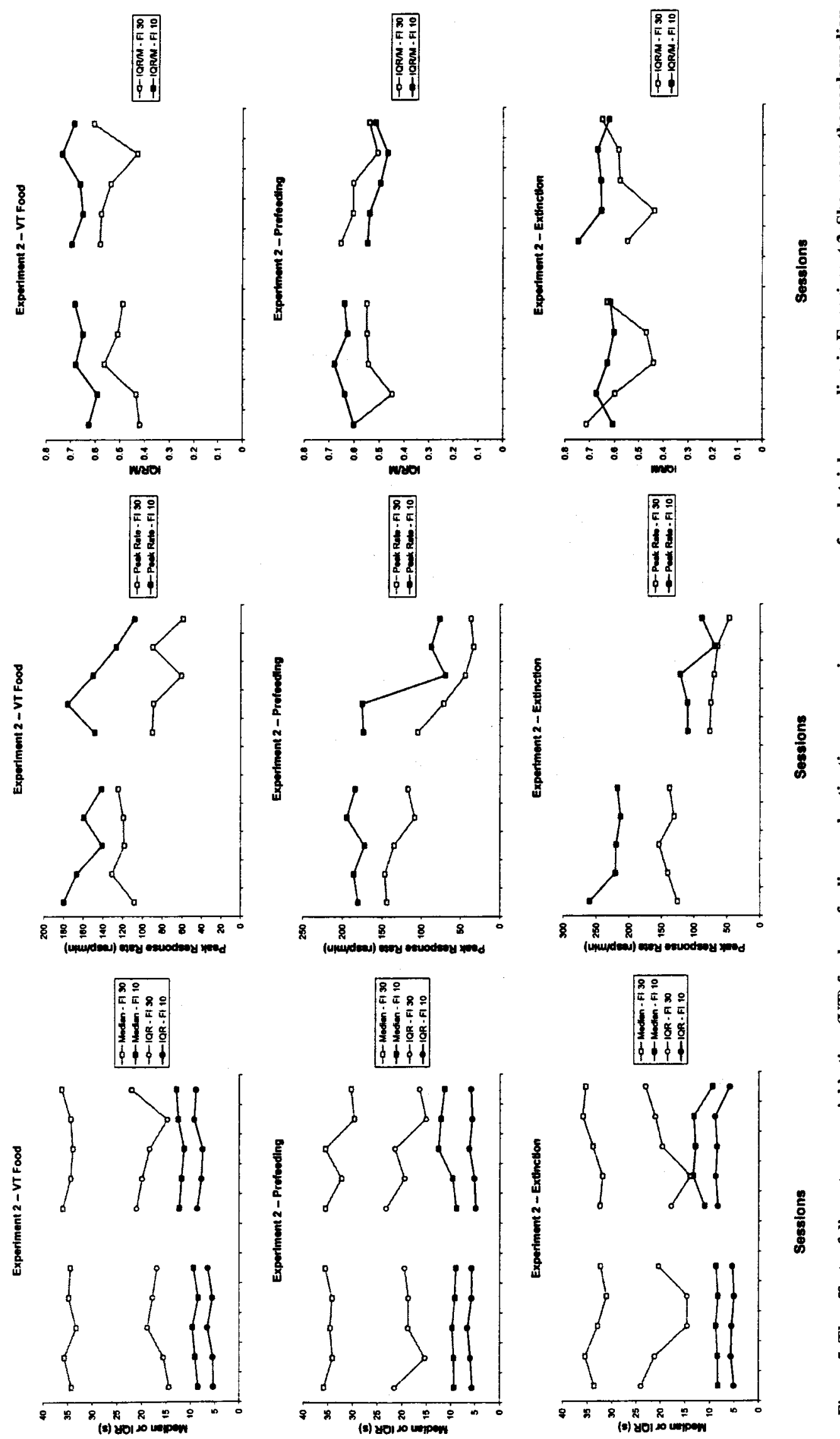
Table 2

Resistance to Change for Experiment 2, Measured as the Log Proportion of Baseline: Results for Overall Response Rate and Four Measures of Performance on Peak Trials

\begin{tabular}{|c|c|c|c|c|c|c|c|c|c|c|}
\hline \multirow[b]{3}{*}{ Disruptor Test } & \multicolumn{10}{|c|}{ Measure } \\
\hline & \multicolumn{2}{|c|}{ Overall Response Rate } & \multicolumn{2}{|c|}{ Median } & \multicolumn{2}{|c|}{$i g r$} & \multicolumn{2}{|c|}{ Peak Rate } & \multicolumn{2}{|c|}{$\mathrm{iqr} / \mathrm{m}$} \\
\hline & Average & $S E$ & Average & $\overline{S E}$ & Average & $S E$ & Average & $S E$ & Average & $S E$ \\
\hline VT, FI $30 \mathrm{sec}$ & -.07 & .05 & .00 & $.01^{*}$ & .05 & $.02 *$ & -.07 & .11 & .05 & .03 \\
\hline VT, FI $10 \mathrm{sec}$ & -.07 & .06 & .16 & $.04^{*}$ & .18 & $.03^{*}$ & -.05 & .07 & .02 & .03 \\
\hline Prefeeding, FI $30 \mathrm{sec}$ & -.31 & .10 & -.01 & .04 & .07 & $.10^{*}$ & -.40 & .05 & .08 & \\
\hline Prefeeding, FI $10 \mathrm{sec}$ & -.32 & .04 & .02 & .04 & -.04 & $.05^{*}$ & -.22 & .06 & -.06 & .02 \\
\hline Extinction, FI $30 \mathrm{sec}$ & -.41 & .18 & -.01 & $.03^{*}$ & -.04 & $.10^{*}$ & -.33 & .13 & -.04 & .07 \\
\hline Extinction, FI $10 \mathrm{sec}$ & -.49 & .17 & .10 & $.05^{*}$ & .14 & $.04^{*}$ & -.54 & .18 & .04 & .02 \\
\hline
\end{tabular}

Note--igr, interquartile range; iqr/m, interquartile range divided by the median; VT, variable time; FI, fixed interval. Positive values indicate increases, relative to baseline; negative values indicate decreases. The data are averaged across subjects. Pairs of values followed by asterisks were significantly different $(p<.05)$ in a planned comparison.

FI 30-sec component during extinction, suggesting a partial reinforcement extinction effect (PREE; Nevin, 1988).

The BeT predicts that variability of responding on peak trials should have decreased during the VT test and that peak location and variability should have increased during extinction. On average, interquartile range increased in both components (see Figure 5), although these increases did not reach significance. There was a similar small increase, overall, in the interquartile range during extinction, but medians remained approximately constant. Thus, the data offer partial support for the BeT's predictions for extinction, but not for the VT test.

Relative variability $(\mathrm{igr} / \mathrm{m})$ was greater in the FI 10-sec component during baseline and test for VT and extinction (see Figure 5). This result is interesting, because it suggests that temporal sensitivity was greater in the leaner ( $\mathrm{FI} 30-\mathrm{sec}$ ) component, an apparent violation of Weber's law and scalar timing (but not Poisson timing). However, it is inconsistent with the results of Beam et al. (1998), who used a similar multiple peak procedure (with FI 20-sec and FI 40-sec schedules) and obtained data consistent with Weber's law.

For all the subjects, peak location in baseline was less, as a proportion of the schedule value, in the FI 10-sec component. This result is similar to Experiment 1 in that responding peaked relatively earlier in the richer component and is not anticipated by current theories of timing.

\section{EXPERIMENT 3}

In Experiment 3, we explored the effects of reinforcement probability. Food was available for responding on $100 \%$ of the regular trials in the rich component, but on only $33 \%$ of the trials in the lean component. Although reinforcement probability has not been investigated in prior research on resistance to change, there is reason to expect that it should be an effective variable. Spetch and Dunn (1987) studied pigeons' preference in concurrent chains for FI schedules that provided reinforcement on either $100 \%$ or $33 \%$ of the trials and found strong preference for the $100 \%$ schedule as long as the no-food trials on the $33 \%$ alternative were not differentially signaled. Because reinforcement parameters generally have the same effects on preference and resistance to change (Grace \& Nevin, 1997), we expected responding in the rich component to be more resistant to change, again with the possible exception of extinction because the generalization decrement during test for the rich component was greater.

\section{Method}

Subjects. The subjects were 4 different White Carneaux pigeons. They had experience with a variety of experimental tasks, but none involving timing procedures. They were housed as was described in Experiment 1.

Procedure. The procedure was similar to that used in Experiment 1 , except that the schedules differed in terms of reinforcement probability on regular trials. In both the rich and the lean components, responses were reinforced according to an FI 20-sec schedule. Reinforcement consisted of $3 \mathrm{sec}$ of access to grain and was available on $100 \%$ of the regular trials $(27 / 27)$ in the rich component, but on only $33 \%(9 / 27)$ in the lean component. Peak-trial duration was $60 \mathrm{sec}$ for both components. Because 9 out of 36 trials were peak trials, the overall reinforcement probability per trial was $75 \%(27 / 36)$ for the rich component and $25 \%(9 / 36)$ for the lean component. For 2 birds, the center key was lighted red during the lean component and green during the rich component; this assignment was reversed for the other birds. Sessions were generally conducted 7 days per week at approximately the same time.

Baseline training consisted of 42 sessions and was followed by the VT test. During this test, which lasted for 6 sessions, grain magazine presentations (1.67-sec duration) were delivered during the ITI according to a VT $7.5-\mathrm{sec}$ schedule. The subjects then completed 11 baseline sessions prior to the prefeeding test, which lasted for 5 sessions. The subjects were returned to baseline for 18 additional sessions, followed by 9 sessions of extinction.

\section{Results}

Figure 6 shows peak-trial response distributions for the $33 \%$ (left panels) and 100\% (right panels) components, for baseline and each disruptor test. The data are aggregated 
Probability - 33\%

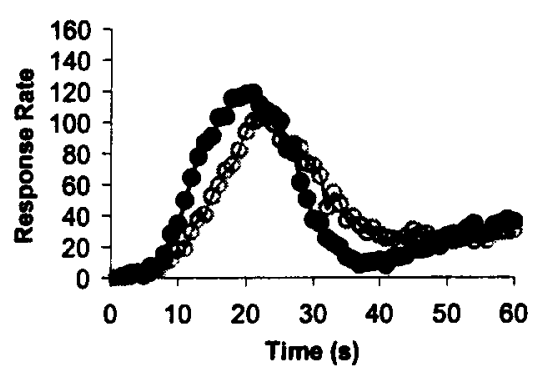

Probability - 33\%

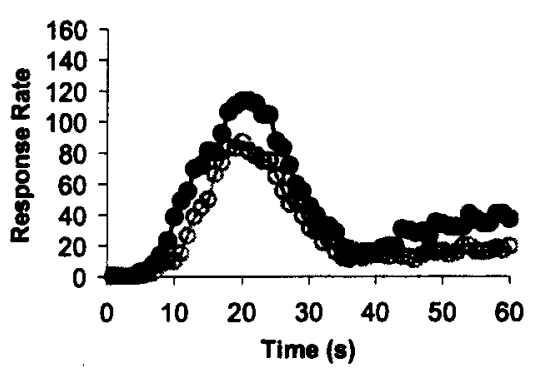

Probability $-33 \%$

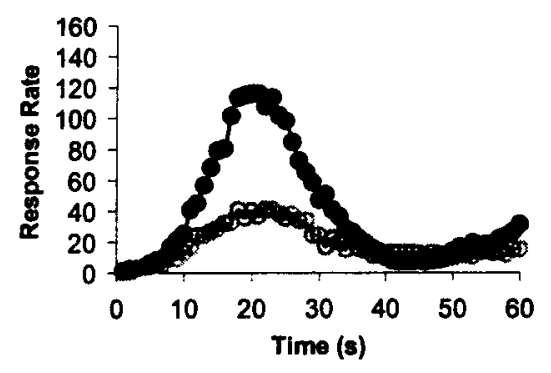

Probability $-100 \%$

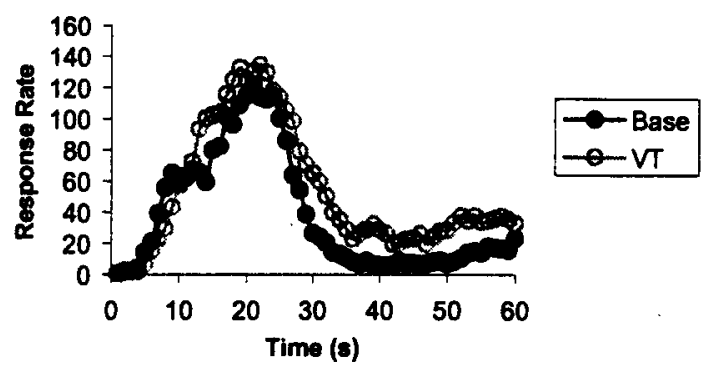

Probability $-100 \%$
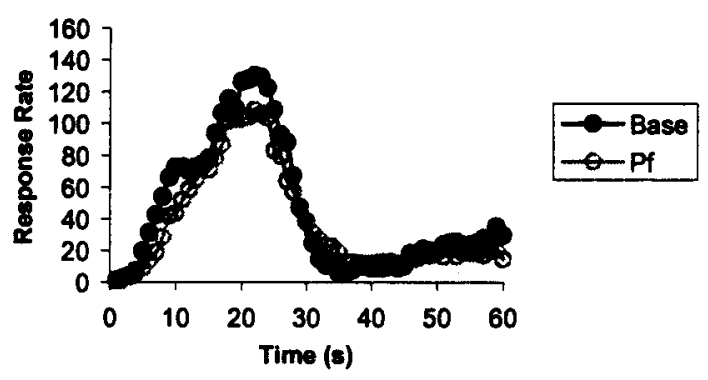
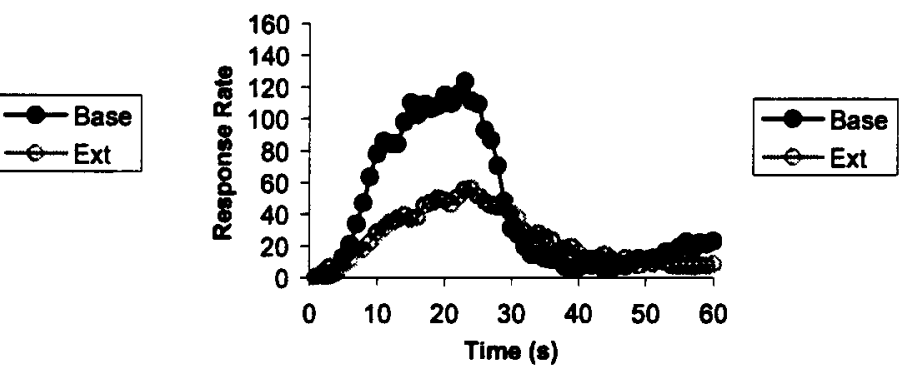

Figure 6. A comparison of peak-trial responding between baseline and the three resistance-to-change tests from Experiment 3. In each panel, the filled circles represent responding on the five baseline sessions preceding a resistance test, and the unfilled circles show responding on the first five sessions of the resistance test. The data from the variable-time (VT), prefeeding, and extinction tests are shown in the upper, middle, and lower panels, respectively. The results from the $33 \%$ component are displayed in the left column; the results from the $100 \%$ component are displayed in the right column. The data are averaged across subjects.

across the last five baseline sessions and the first five sessions of each disruptor test and are averaged across subjects. The only significant difference between the components in baseline is that the location of the peak (i.e., median) occurred earlier in the $100 \%$ component $(M=$ $19.04)$ as compared with the $33 \%$ component $[M=20.37$; $F(1,3)=35.66]$. During the prefeeding and extinction tests, responding generally decreased but remained at the same level or even increased during the VT test.

For a more detailed assessment of disruptor effects, single-session data are presented in Figure 7 (left pan- els: median and interquartile range; center panels: peak response rate; right panels: iqr $/ m$ ). The data represent the last five sessions of each pretest baseline and the first five sessions of each disruptor test and are averaged across subjects. Individual data were entered into a series of repeated measures ANOVAs, with type of disruptor, component (33\% or $100 \%$ ), type of session (baseline/test), and session as factors. For the medians, the effect of disruptor type was significant $[F(1,3)=16.51]$, as were the session type $\times$ session $[F(8,24)=3.53]$ and disruptor type $\times$ session $[F(4,12)=3.52]$ interactions. Post hoc 

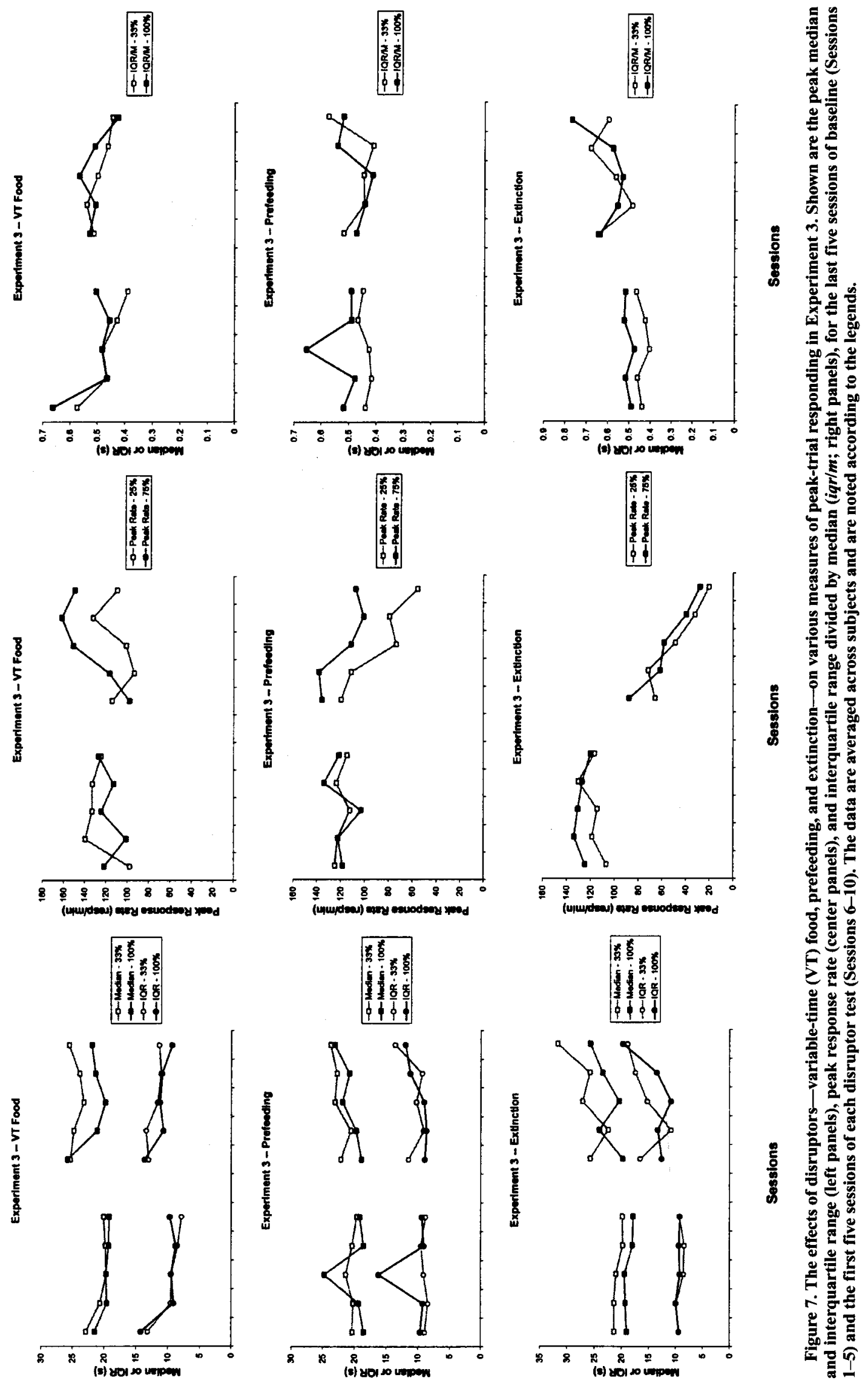

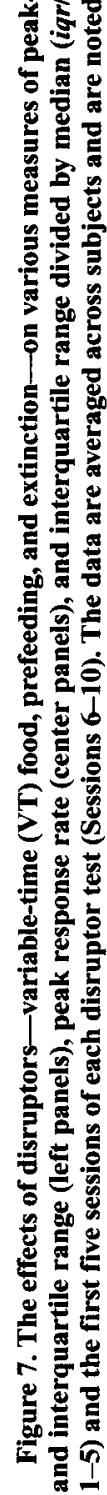


Table 3

Resistance to Change for Experiment 3, Measured as the Log Proportion of Baseline: Results for Overall Response Rate and Four Measures of Performance on Peak Trials

\begin{tabular}{|c|c|c|c|c|c|c|c|c|c|c|}
\hline \multirow[b]{3}{*}{ Disruptor Test } & \multicolumn{10}{|c|}{ Measure } \\
\hline & \multicolumn{2}{|c|}{ Overall Response Rate } & \multicolumn{2}{|c|}{ Median } & \multicolumn{2}{|c|}{$i q r$} & \multicolumn{2}{|c|}{ Peak Rate } & \multicolumn{2}{|c|}{$i q r / m$} \\
\hline & Average & $S E$ & Average & $S E$ & Average & $S E$ & Average & $S E$ & Average & $S E$ \\
\hline VT, $33 \%$ & -.12 & .05 & .09 & .03 & .15 & .02 & -.09 & $.01 *$ & .06 & .03 \\
\hline VT, $100 \%$ & .07 & .05 & .04 & .02 & .09 & .05 & .06 & $.04^{*}$ & .05 & .05 \\
\hline Prefeeding, $33 \%$ & -.18 & .07 & .03 & .01 & .05 & .04 & -.16 & .07 & .02 & .05 \\
\hline Prefeeding, $100 \%$ & -.08 & .05 & .03 & .01 & .01 & .03 & -.06 & .10 & -.01 & .04 \\
\hline Extinction, $33 \%$ & -.43 & .11 & .04 & .04 & .14 & .05 & -.45 & .07 & .10 & .01 \\
\hline Extinction, $100 \%$ & -.35 & .07 & .07 & .01 & .14 & .03 & -.45 & .09 & .07 & .04 \\
\hline
\end{tabular}

Note-iqr, interquartile range; iqr $/ m$, interquartile range divided by the median; VT, variable time. Positive values indicate increases, relative to baseline; negative values indicate decreases. The data are averaged across subjects. Pairs of values followed by asterisks were significantly different $(p<.05)$ in a planned comparison.

comparisons found that the increases in the median for the $33 \%$ component reached significance during extinction. For interquartile range, the main effect of disruptor type was significant $[F(1,3)=16.21]$. Post hoc comparisons found that interquartile range increased significantly in both components during extinction. For peak response rate, the main effect of session type $[F(2,6)=$ $5.70]$ and the session type $\times$ disruptor type interaction $[F(2,6)=6.76]$ reached significance. Post hoc comparisons found that the decreases in peak response rate in both components were significant during extinction. For the temporal sensitivity $($ iqr $/ m$ ) data in Figure 7, the session type $\times$ disruptor and the session type $\times$ component $\times$ session interactions reached significance $[F(2,6)=$ 5.13 and $F(8,24)=3.12$, respectively]. Post hoc comparisons found that iqr/m increased in the $33 \%$ component during extinction.

We computed resistance to change as the log proportion of baseline for overall response rate and for measures of performance on peak trials (median, interquartile range, peak response rate, and $i q r / m)$. The results were based on data aggregated over the five baseline and five test sessions contributing to Figure 6. Average resistance-to-change values are listed in Table 3. Individual data were analyzed with repeated measures ANOVAs, with type of disruptor and component $(33 \%$ or $100 \%)$ as factors. For $\log$ proportion of overall response rate, there was a significant effect of disruptor type $[F(2,6)=5.92]$; extinction decreased responding more than did the other two disruptors. However, none of the differences between the $33 \%$ and the $100 \%$ components reached significance. For log proportion of peak response rate, there was a significant effect of disruptor type $[F(2,6)=$ 7.12], similarly because extinction had a greater decremental effect on responding than did the other disruptors. For all the subjects, resistance to change was greater in the $100 \%$ component during the VT test. There were no significant effects on resistance-to-change measures derived from the median, interquartile range, or $i q r / m$, although with the latter measure for all 4 subjects resis- tance to change was less in the $33 \%$ component during prefeeding.

\section{Discussion}

Behavioral momentum theory predicts that responding in the $100 \%$ component should be more resistant to change during the VT and prefeeding tests; differential generalization decrement in extinction is expected to counteract the strengthening effects of more frequent reinforcement in the $100 \%$ component. As in Experiments 1 and 2, the results were mixed when resistance to change was measured as the $\log$ proportion of overall response rate. Although, on average, resistance to change was greater in the $100 \%$ component for all the disruptor tests, there was at least one reversal among the subjects in each test, and none of the planned comparisons reached significance. For log proportion of peak response rate, the results were somewhat clearer: For all 4 subjects, responding decreased more in the $33 \%$ component during the VT test. On average, peak responding decreased more during prefeeding but did not reach significance, because the data for 1 subject showed no difference. There were no systematic differences in resistance to extinction. With resistance to change assessed by changes in temporal control, on average, $i q r / m$ increased more in the $33 \%$ component in all the disruptor tests (indicating that temporal control was more disrupted) and was obtained for all 4 subjects in prefeeding. Overall, these results show more betweensubjects variability than in the first two experiments, but the peak response rate and iqr/m data are reasonably in accord with expectations based on momentum theory.

Were the changes in the peak response distributions during the disruptor tests consistent with the BeT? Peak location (i.e., median) increased in the $33 \%$ component during extinction, as did variability (i.e., interquartile range) in both components. These upward shifts in peak location and variability are predicted by the BeT. However, we found no evidence of decreases in variability 
during the VT or the prefeeding tests; if anything, variability tended to increase. As in Experiment 1, these data provide partial support for the BeT.

\section{GENERAL DISCUSSION}

Our primary goal was to determine whether a more effective measure of response strength, in terms of resistance to change, could be obtained for FI schedules, using the peak procedure. Typically, resistance to change has been measured as the log proportion of baseline response rate. This measure works well for VI schedules, which maintain steady responding, but may not be appropriate for FI schedules, in which responding is characterized by temporal control.

We examined various measures of performance in a multiple peak procedure with differential reinforcement arranged in three ways: reinforcement magnitude (Experiment 1), immediacy (Experiment 2), and probability (Experiment 3). In addition, we arranged three resistance tests in each experiment (intercomponent food delivered according to a VT schedule, prefeeding, and extinction). The prediction of momentum theory is that responding in the component with the relatively greater magnitude, immediacy, or probability of reinforcement should be more resistant to change. In particular, we hypothesized that changes in peak response rate (on peak trials only) would prove more sensitive to differential reinforcement than would changes in overall response rate and thus would provide a more appropriate measure of resistance to change for FI schedules.

How well were the predictions of momentum theory supported, given the different measures of resistance to change? We will first deal with the VT and prefeeding test data. With log proportion of overall response rate as the measure of resistance to change and pooling across experiments and subjects, in the VT tests the richer component was more resistant to change in 6 out of $12 \mathrm{com}-$ parisons $[t(11)=-1.70, n . s$.$] . In the prefeeding tests$ with the same measure, the richer component was more resistant to change in 6 out of 12 comparisons $[t(11)=$ -0.79, n.s.]. Overall, there were no systematic differences across experiments in the VT and prefeeding tests, using the traditional measure of resistance to change.

Turning now to $\log$ proportion of peak response rate, in the VT tests the richer component was more resistant to change in 9 of 12 comparisons, and the difference was significant $[t(11)=-2.26]$. For prefeeding, the richer component had greater resistance in 11 out of $12 \mathrm{com}-$ parisons and also reached significance $[t(11)=-4.93]$. Thus, the prediction of momentum theory was supported when resistance to change was measured as change in peak response rate relative to baseline.

One other measure of change in the disruptor tests appears to hold some promise: log proportion of change in temporal sensitivity, measured as the interquartile range divided by the median (iqr/m; a nonparametric analogue to the coefficient of variation). Because smaller values of iqr $/ m$ indicate greater relative temporal control and $\mathrm{iqr} / \mathrm{m}$ increased or remained about the same during resistance tests, a relatively smaller increase in the richer component would indicate greater response strength. In the VT tests, the richer component was more resistant to change in 8 out of 12 comparisons $[t(11)=1.53$, n.s.]. For prefeeding, the richer component was more resistant in 12 out of 12 comparisons, and the difference was significant $[t(11)=3.94]$. Although the VT results did not reach significance, the prefeeding data suggest that reinforcement strengthens the temporal control of responding, as measured by resistance to change.

When components differ in terms of reinforcement rate (Experiment 2) or probability (Experiment 3), extinction is confounded as a test of resistance to change, because of generalization decrement; the stimulus situation changes more in the richer component during test, which may counteract any effects of reinforcement on response strength. However, this confound should not apply to reinforcement magnitude. Indeed, in Experiment 1, the richer component was more resistant to extinction, as measured by changes in both overall and peak response rates (see Table 1). In contrast, the data from Experiment 2 show a PREE-type effect, with peak response rate in the richer (FI 10-sec) component being less resistant to extinction for all 4 subjects (see Table 2). Extinction tests in Experiment 3 were mixed, with 2 of 4 subjects showing greater resistance in the richer component with both overall and peak response rate measures. Thus, the extinction data from Experiment 1 provide some confirmation for predictions of momentum theory. The PREE-like results in Experiments 2 and 3 are consistent with an augmented model of resistance to extinction that incorporates generalization decrement proposed by Nevin and Grace (1999; see also Nevin \& Grace, 2000).

The results were most consistent with the prefeeding test, somewhat less so with VT (Experiments 1 and 3 successful), whereas extinction produced two clear cases in which the richer component was not more resistant to change (Experiments 2 and 3 ). It makes sense that the results of the prefeeding test would be the strongest, because it is the least confounded resistance test, in the sense that only the subjects' internal state is affected, with no change whatsoever to the session contingencies. In the VT test, on the other hand, although the baseline schedules remain in place, food is delivered during the ITI, which could affect responding directly.

A good criterion for a measure of relative response strength in FI schedules is that it be maximally sensitive to differential reinforcement. We have measured relative response strength as the difference in log proportion of baseline, $\log \left(B_{x 1} / B_{o 1}\right)-\log \left(B_{x 2} / B_{o 2}\right)$ (Grace \& Nevin, $1997)$, plugging in different measures of behavior. Because this measure may be positive or negative (i.e., positive in the case of the richer component's being more resistant to change, but negative for the PREE-type effects in Experiment 2), we can compare the sensitivity of the various measures to differential reinforcement by taking 
absolute values. Pooling across all resistance tests and experiments, the absolute value of relative resistance to change, using peak response rate, was significantly greater $(M=0.184)$, as compared with overall response rate $[M=$ $.135 ; t(35)=2.31]$. This provides evidence in support of our hypothesis that peak response rate would provide a more effective measure of resistance to change in FI schedules than would overall response rate, because it is more sensitive to differential reinforcement.

Log proportion of peak, rather than overall response rate, appears to be a more appropriate measure of resistance to change from theoretical considerations as well. Because of the temporal control evident in FI responding (Zeiler \& Powell, 1994), peak response rate is that portion of behavior with the greatest temporal contiguity to reinforcement. Since temporal contiguity is crucial for Pavlovian conditioning and Nevin, Tota, Torquato, and Shull (1990) have shown that resistance to change depends primarily on Pavlovian contingencies, it is reasonable to expect that peak response rate will provide a maximally sensitive assay of resistance to change for FI schedules.

Measuring resistance to change in FI schedules as log proportion of peak response rate may help to address some anomalies in the literature. Cohen et al. (1993) noted that resistance to change in FI schedules seemed less sensitive than that for comparable VI schedules. However, their results were based on measuring resistance to change in terms of overall response rate. A more difficult problem is Mandell's (1980) finding that average response rates on FI and VI schedules that delivered the same overall reinforcement rate did not differ in resistance to change. Because subjects strongly prefer VI to FI schedules when arranged as the terminal links in concurrent chains (Herrnstein, 1964; Killeen, 1968), Mandell's results constitute a major counterinstance to the general covariation of resistance to change and preference. It is possible that a parametric investigation of this issue, using a variant of the peak procedure so that peak response rate may be used as a measure of resistance to change, may help to resolve the discrepancy.

If reinforcement strengthens not only peak response rate, but also the temporal control of responding, measures of resistance to change based on peak rate and temporal sensitivity (igr/m) ought to be correlated. Specifically, the correlation should be negative, because iqr $/ m$ tended to increase, rather than decrease, during the resistance tests and smaller increases are indicative of greater strength. To test this hypothesis, we computed correlations between the five measures of resistance to change: log proportions of peak response rate, temporal sensitivity $(\mathrm{iqr} / \mathrm{m})$, median, interquartile range, and overall response rate. There was a total of 72 values on each variable entering into the correlations (for each experiment: 4 subjects $\times 3$ resistance tests $\times 2$ components). As was predicted, there was a significant negative cor- relation between log proportion of peak response rate and $i q r / m(r=-.26)$. This is not artifactual, since peak rate and temporal sensitivity are independent measures that can be experimentally dissociated (Roberts, 1981). (Note that the correlation between resistance to change measured as overall response rate and $\mathrm{iqr} / \mathrm{m}$ was close to zero; $r=.05, \mathrm{n} . \mathrm{s}$.) This negative correlation is consistent with our assumption that change in peak rate and temporal sensitivity are independent expressions of a single construct representing response strength, or behavioral mass. Correlations between $\log$ proportions of peak and overall response rate and median and interquartile range were both strongly positive ( $r \mathrm{~s}=.89$ and .69 , respectively). The latter may represent a Weber's law-type relationship. Overall, the structure of the resistance data provides support for the notion that reinforcement strengthens both the peak level and the temporal control of responding under FI schedules.

Our secondary goal was to ask whether changes in behavior that occurred during the disruptor tests were consistent with current theory. Of the current theories of animal timing, only Killeen and Fetterman's (1988) BeT (see also Machado, 1997) makes clear predictions for the disruptor tests.

According to the BeT, pacemaker speed varies directly with the overall rate of reinforcement. When that is changed through addition of VT food, extinction, or prefeeding (which may be construed as an increase in reinforcement rate external to the session), pacemaker speed changes, resulting in changes in peak location and variability according to Equations 1 and 2 . Thus, the BeT predicts that peak location and variability should increase during extinction (Killeen et al., 1999). For VT food and prefeeding, pacemaker speed should increase, leading to decreases in peak location and variability. The decrease in location should be only temporary, however, since animals should recalibrate their behavior as long as reinforced trials are still available. Thus, the BeT predicts that peak variability, but not location, should show durable decreases during VT and prefeeding (Fetterman \& Killeen, 1995).

Were these predictions confirmed? For extinction, the only significant changes for median (peak location) and interquartile range (variability) were increases (Experiments 1 and 3). This confirms the BeT's prediction. However, the only significant changes during the prefeeding and VT tests for these measures were also increases (Experiment 1). We found no evidence that peak variability decreased upon initial exposure to the VT or the prefeeding disruptors. Interestingly, peak variability did decrease to below-baseline levels in Experiment 1 when the VT test continued for 20 sessions (see Figure 3). But it is uncertain whether this pattern of an initial increase followed by a decrease with extended training can be reconciled with the BeT. The sudden imposition of VT food should have produced an immediate decrease, fol- 
lowed, perhaps, by a return to baseline levels through recalibration. Thus, the present data can offer only partial support for the BeT.

Our final question was whether reliable differences in peak-trial responding in baseline would be observed. There is no basis for expecting any differences in terms of timing theories. Yet in all three experiments, peak location occurred earlier in the richer component, relative to the FI schedule value. The effect was small, averaging a difference of just $1.58 \mathrm{sec}$ in Experiments 1 and 3 . However, it was consistent across subjects, and the fact that it was obtained with three different manipulations of differential reinforcement provides fairly strong converging evidence. The question is why it would occur. According to the BeT, pacemaker speed should be faster in the richer component, but performances should recalibrate so that there would be no difference between the peak medians. One possibility is that recalibration is incomplete and does not fully compensate for the faster pacemaker speed in the rich component. The earlier peak in the rich component is consistent with a faster pacemaker in that component.

There might be evidence for incomplete recalibration if the peak medians were increasing in the rich component at the end of baseline training. To examine this possibility, we computed regression slopes across the last 10 baseline sessions prior to the VT test in all the experiments. However, regression slopes in both rich $(M=$ $-0.12)$ and lean $(M=-0.17)$ components were not systematically different from zero (both n.s.), suggesting that performances were stable. Thus, the earlier peak location in the rich components remains an anomaly for future research to explore.

Overall, our research demonstrates that a multiple peak procedure with tests of resistance to change provides orderly single-session data and can be used simultaneously to address questions relevant to behavioral momentum and temporal stimulus control. It has identified two measures-change in peak response rate and temporal sensitivity (iqr/m) - that may be more appropriate measures of response strength for FI schedules than is change in overall response rate. Future research can use this procedure to examine other issues, such as acquisition of temporal control (i.e., changes in peaktrial responding when FI schedules are altered), which will help to develop more comprehensive theories of timing and response strength.

\section{REFERENCES}

Beam, J. J., Killeen, P. R., Bizo, L. A., \& Fetterman, J. G. (1998). How reinforcement context affects temporal production and categorization. Animal Learning \& Behavior, 26, 388-396.

Catania, A. C. (1970). Reinforcement schedules and psychophysical judgments: A study of some temporal properties of behavior. In W. N. Schoenfeld (Ed.), The theory of reinforcement schedules (pp. 1-42). New York: Appleton-Century-Crofts.

Cheng, K., \& RoBerTs, W. A. (1991). Three psychophysical principles of timing in pigeons. Learning \& Motivation, 22, 112-128.
Cohen, S. L., Riley, D. S., \& Weigle, P. A. (1993). Tests of behavior momentum in simple and multiple schedules with rats and pigeons. Journal of the Experimental Analysis of Behavior, 60, 255-291.

FERSTER, C. B., \& SKINNER, B. F. (1957). Schedules of reinforcement. New York: Appleton-Century-Crofts.

Fetterman, J. G., \& Killeen, P. R. (1991). Adjusting the pacemaker. Learning \& Motivation, 22, 226-252.

Fetterman, J. G., \& KilleEN, P. R. (1995). Categorical scaling of time: Implications for clock-counter models. Journal of Experimental Psychology: Animal Behavior Processes, 21, 43-63.

Fleshler, M., \& Hoffman, H. S. (1962). A progression for generating variable-interval schedules. Journal of the Experimental Analysis of Behavior, 5, 529-530.

GiBвoN, J. (1977). Scalar expectancy theory and Weber's law in animal timing. Psychological Review, 84, 279-325.

Grace, R. C., \& Nevin, J. A. (1997). On the relation between preference and resistance to change. Journal of the Experimental Analysis of Behavior, 67, 43-65.

Grace, R. C., \& Nevin, J. A. (1999). Timing and choice in concurrent chains. Behavioural Processes, 45, 115-127.

HARPER, D. N. (1996). Response-independent food delivery and behavioral resistance to change. Journal of the Experimental Analysis of Behavior, 65, 549-560.

HARPER, D. N., \& MCLEAN, A. P. (1992). Resistance to change and the law of effect. Journal of the Experimental Analysis of Behavior, 57, 317-337.

HeRRNSTEIN, R. J. (1964). Aperiodicity as a factor in choice. Journal of the Experimental Analysis of Behavior, 7, 179-182.

KILLEEN, P. (1968). On the measurement of reinforcement frequency in the study of preference. Journal of the Experimental Analysis of Behavior, 11, 263-269.

KilleEn, P. R., \& FetTERman, J. G. (1988). A behavioral theory of timing. Psychological Review, 94, 274-285.

Killeen, P. R., \& Fetterman, J. G. (1993). The behavioral theory of timing: Transition analyses. Journal of the Experimental Analysis of Behavior, 59, 411-422.

KilleEN, P. R., HALL, S., \& Bizo, L. A. (1999). A clock not wound runs down. Behavioural Processes, 45, 129-139.

MACEwEn, D., \& KiLleEN, P. (1991). The effects of rate and amount of reinforcement on the speed of the pacemaker in pigeons' timing behavior. Animal Learning \& Behavior, 19, 164-170.

MACHADO, A. (1997). Learning the temporal dynamics of behavior. Psychological Review, 104, 241-265.

MANDELL, C. (1980). Response strength in multiple periodic and aperiodic schedules. Journal of the Experimental Analysis of Behavior, 33, 221-241.

Morgan, L., Killeen, P. R., \& Fetterman, J. G. (1993). Changing rates of reinforcement perturbs the flow of time. Behavioural Processes, 30, 259-272.

NEVIN, J. A. (1974). Response strength in multiple schedules. Journal of the Experimental Analysis of Behavior, 21, 389-408.

Nevin, J. A. (1988). Behavioral momentum and the partial reinforcement effect. Psychological Bulletin, 103, 44-56.

Nevin, J. A., \& GRACE, R. C. (1999). Does the context of reinforcement affect resistance to change? Journal of Experimental Psychology: Animal Behavior Processes, 25, 256-268.

Nevin, J. A., \& Grace, R. C. (2000). Behavioral momentum and the law of effect. Behavioral \& Brain Sciences, 23, 73-130. [Includes commentary]

Nevin, J. A., Mandell, C., \& Atak, J. R. (1983). The analysis of behavioral momentum. Journal of the Experimental Analysis of Behavior, 39, 49-59.

Nevin, J. A., Mandell, C., \& Yarensky, P. (1981). Response rate and resistance to change in chained schedules. Journal of Experimental Psychology: Animal Behavior Processes, 7, 278-294.

Nevin, J. A., Smith, L. D., \& Roberts, J. (1987). Does contingent reinforcement strengthen operant behavior? Journal of the Experimental Analysis of Behavior, 48, 17-33.

Nevin, J. A., Tota, M. E., Torquato, R. D., \& Shull, R. L. (1990). Alternative reinforcement increases resistance to change: Pavlovian or 
operant contingencies? Journal of the Experimental Analysis of Behavior, 53, 359-379.

ROBERTS, S. (1981). Isolation of an internal clock. Journal of Experimental Psychology: Animal Behavior Processes, 7, 242-268.

Shettleworth, S., \& Nevin, J. A. (1965). Relative rate of response and relative magnitude of reinforcement in multiple schedules. Journal of the Experimental Analysis of Behavior, 8, 199-202.

SPEtch, M. L., \& DunN, R. (1987). Choice between reliable and unreliable outcomes: Mixed percentage-reinforcement in concurrent chains. Journal of the Experimental Analysis of Behavior, 47, 57-72.

ZeIler, M. D., \& Powell, D. G. (1994). Temporal control in fixedinterval schedules. Journal of the Experimental Analysis of Behavior, 61, 1-9.

\section{NOTE}

1. We assessed the comparability of the median and interquartile range with parameters recovered from normal distributions as measures of peak location and spread. Normal distributions (with a scale param- eter for response rate) were fitted to the baseline and the test data for each disruptor (aggregated over the five sessions). In general, Gaussian functions fitted the distributions well; the median percentage of variance accounted for was .91 for the small-magnitude component and .92 for the large-magnitude component. The means and standard deviations were then compared against the nonparametrics from the same sessions. The correlations between the means and medians, standard deviations and interquartile ranges, and recovered heights of the normal distributions and peak response rates were all greater than .90 . This suggests that to a fair approximation, the nonparametrics were linearly related to the parameters from fitted normal distributions, and this conclusion was supported by visual inspection of scatterplots. Results of similar analyses performed on the data from Experiments 2 and 3 were also consistent with this view.

(Manuscript received July 6, 1999 revision accepted for publication May 22,2000 .) 